\title{
SUR LA VOIE DE L'AUTOMOBILE AUTONOME Ma voiture et comment elle voit le monde
}

\author{
José BRETES \\ Laser Components \\ j.bretes@lasercomponents.fr
}

Dans le monde de la science-fiction, les voitures autonomes sont pratiquement des véhicules standards. Dans la « vraie vie ", nous rattrapons rapidement l'imagination des auteurs. Aujourd'hui encore, il semble qu'un nouveau système d'alerte soit ajouté chaque année. Des systèmes d'alerte de changement de voie et des assistants de distance et de stationnement sont déjà disponibles dans les voitures de milieu de gamme. Les modèles les plus chers se conduisent pratiquement tout seuls, au moins en mode stop-and-go. Est-ce à dire que les banlieusards ont le temps de faire une petite sieste dans les embouteillages quotidiens du matin ? La technologie n'en est pas encore tout à fait là, mais il existe une réelle concurrence entre les constructeurs automobiles sur la voie de la conduite autonome.

Elles émettent des ondes ultrasonores et peuvent reconnaître les proies et les obstacles au moyen de l'écho réfléchi. Leur portée maximale est d'environ douze mètres. Les capteurs à ultrasons utilisés dans les voitures fonctionnent selon le même principe. Le problème est que les ondes sonores générées artificiellement ont également une portée relativement courte ; elles ne peuvent donc être utilisées qu'à courte distance. Les applications les plus connues sont les télémètres pour les aides au stationnement.

Les premiers modèles avec ce système d'assistance sont apparus sur le marché au début des années 1980. La technologie à ultrasons n'est plus seulement utilisée à l'arrière de la voiture, mais aussi avec des capteurs d'angle mort ou pour mesurer la distance du véhicule qui précède à basse vitesse. En plus de la courte portée, les ingénieurs doivent également tenir compte des facteurs d'interférence externes lorsqu'ils mettent au point des systèmes d'assistance à base d'ultrasons. Les freins hydrauliques des camions et des autobus, par exemple, génèrent également des ondes ultrasonores, qui peuvent provoquer une confusion acoustique avec les capteurs situés à proximité immédiate.

\section{Une voiture avec des yeux}

Les systèmes de caméras sont probablement ceux qui imitent le plus la perception humaine. Une caméra installée dans la voiture enregistre en permanence l'environnement du véhicule pendant le trajet. Le logiciel interprète les données. Il reconnaît, par exemple, les arêtes qui pourraient signifier d'autres véhicules ou des marques de voie. Comme la résolution des caméras augmente continuellement, de plus en plus de détails peuvent être évalués. Cela permet au système de détecter non seulement les obstacles, mais aussi les panneaux de signalisation ou les feux de signalisation. Cette information aide à prévenir les accidents et contribue à l'orientation, car la caméra reconnaît également les détails qui ne sont pas enregistrés sur 


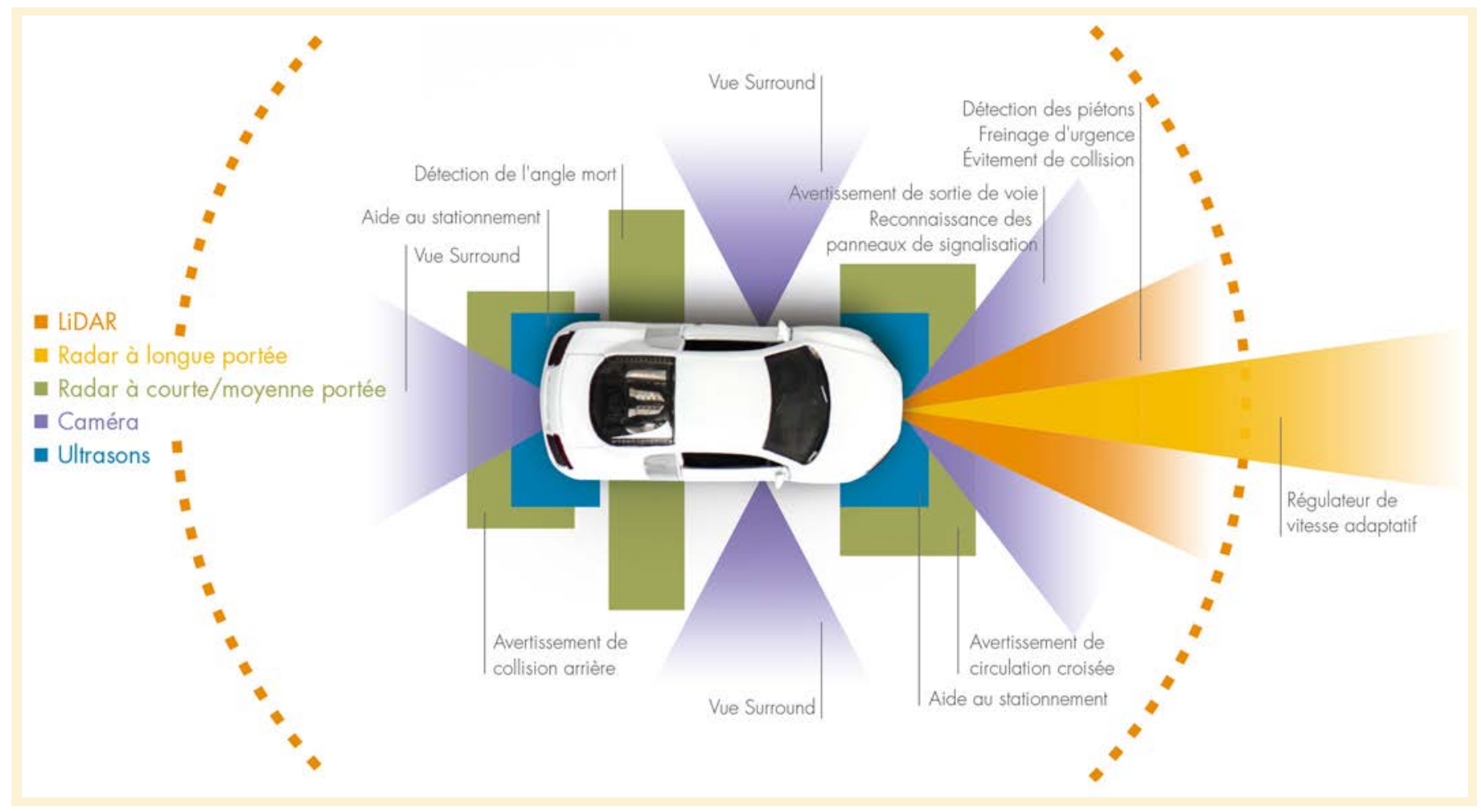

de quelques nanosecondes et - comme nous le savons tous - rien n'est plus rapide que la lumière, le LiDAR fournit des informations fiables dans les plus brefs délais. Comparé à d'autres systèmes, le champ de balayage d'une seule unité émetteur-récepteur est toutefois limité.

À la recherche d'un champ de vue optimal, différents systèmes de balayage, mécaniques ou à l'état solide, sont en cours d'évaluation.

Les systèmes de balayage mécaniques utilisent un mécanisme rotatif, des miroirs galvo ou des MEMs tandis que les systèmes à l'état solide utilisent des guides d'ondes, des réseaux phasés ou des méta-surfaces. Il existe quelques nouveaux systèmes utilisant la même méthode FMCW que le radar pour obtenir des informations de vitesse et de distance dans un signal optique de retour.

La méthode LiDAR fonctionne beaucoup plus rapidement que la méthode radar et fournit une plus grande quantité de données précises. Pourtant, la conception de l'instrument LiDAR doit tenir compte des limites atmosphériques comme le brouillard, le smog et le rayonnement solaire. Pour résoudre les problèmes de balayage et de conditions atmosphériques, chaque méthode
LiDAR peut optimiser la puissance de sortie du laser, modifier la longueur d'onde du laser et la fréquence du radar, ou utiliser plusieurs réseaux de lasers et de détecteurs. Le résultat final est d'obtenir une détection d'objet à plus de 300 mètres dans toutes les conditions de conduite imaginables.

\section{La force du nombre}

Avec le système automatisé - maintenant connu sous le nom d'autonome - de conduite, rien ne peut être laissé au hasard. Alors qu'un être humain peut intuitivement s'appuyer sur sa riche expérience et réagir intuitivement à différentes situations, un ordinateur doit constamment prendre de nouvelles décisions. Pour ce faire, il a besoin d'autant de données que possible. Chaque système de capteurs peut contribuer à cette décision avec ses avantages et ses inconvénients spécifiques. Il existe des prototypes qui ne sont équipés que de caméras ou exclusivement de LiDAR. La plupart des constructeurs automobiles font toutefois appel à des solutions dans lesquelles plusieurs des technologies susmentionnées sont utilisées simultanément. Cette fusion leur permet d'exploiter au mieux les forces de chaque procédé et de bénéficier d'effets synergiques.

\section{L'ÉMETTEUR ET RÉCEPTEUR À PARTIR D'UNE SEULE RESSOURCE}

Les systèmes LiDAR doivent être à la fois fiables, petits et rentables. Pour les fabricants d'appareils de mesure laser et de composants optoélectroniques, il s'agit d'un grand défi. Laser Components fabrique tous les composants pour des solutions LiDAR puissantes et orientées vers l'avenir dans ses installations de production certifiées ISO : des diodes laser pulsées à impulsions ultra-courtes offrent une meilleure résolution pour la mesure de distance. En combinaison avec des photodiodes avalanche très sensibles (APD), même les plus petits signaux peuvent être détectés. En outre, une coopération a été établie avec l'Institut Fraunhofer pour les circuits et systèmes microélectroniques (IMS) pour les barrettes CMOS-SPAD à 1 et 2 dimensions. Les chercheurs de Duisburg peuvent apporter de nouvelles technologies de capteurs qui promettent des mesures particulièrement précises. 